\title{
Assessment of Genetic Variability, Character Association and Path Coefficient of Some Quantitative Traits of Chilli
}

\author{
Dipak Kumar Murmu ${ }^{1}$, Bimal Das ${ }^{2 *}$, Rakesh Yanzone $^{3}$ and Ratul Barman ${ }^{4}$ \\ ${ }^{1}$ Department of Vegetable and Spice Crops, UBKV, RRS, Majhian, India \\ ${ }^{2}$ Department of Genetics and Plant Breeding, College of Agriculture, Majhian, UBKV \\ (Extended Campus), Majhian, India \\ ${ }^{3}$ Department of Plant Pathology, College of Agriculture, Majhian, UBKV \\ (Extended Campus), Majhian, India \\ ${ }^{4}$ Department of Genetics and Plant Breeding, UBKV, RRS, Majhian, India \\ *Corresponding author
}

\section{A B S T R A C T}

\begin{tabular}{|l|}
\hline Ke y w or d s \\
$\begin{array}{l}\text { Chilli (Capsicum } \\
\text { annuum L), Genetic } \\
\text { variability, Fruit } \\
\text { weight, Number of } \\
\text { fruits plant. }\end{array}$ \\
\hline Article Info \\
\hline $\begin{array}{l}\text { Accepted: } \\
\text { 20 February } 2017 \\
\text { Available Online: } \\
\text { 10 March } 2017\end{array}$ \\
\hline \hline
\end{tabular}

Chilli (Capsicum annuum L) is one of the most important cum vegetable spice crop grown in India with great export potential. The exoeriment was undertaken to study the variability, genetic correlations and path coefficients of yielding traits in 24 genotypes of chilli. The analysis of variance revealed the significant differences among the genotypes for almost all the characters studied which indicating that presence of great deal of genetic variability for different traits. Among these genotype Hyb-3(2)-2 one of the most promising one showed maximum fruit yield plant $^{-1}$, fruit length, fruit girth and pericarp thickness. Genetic variability of fruit yield plant ${ }^{-1}$, number of fruits plant ${ }^{-1}$, fruit girth and seeds fruit ${ }^{-1}$ emerged as most reliable characters for selection because of their probable conditioning by the additive gene action. Moderate GCV coupled with high broad sense heritability and moderately high genetic advance was registered in three characters namely fruit yield plant ${ }^{-1}$, number of fruits plant ${ }^{-1}$, fruit girth and seeds fruit ${ }^{-1}$. Character associations both correlation and path co-efficient revealed that fruit weight, number of fruits plant ${ }^{-1}$, primary branches plant ${ }^{-1}$ and plant canopy were the most important selection criteria for improving yield of both green and dry chilli.

\section{Introduction}

Chilli (Capsicum annuum L.) mainly used for its pungency and pleasant flavor and one of the most important vegetable and condiment crop having immense commercial and therapeutic value. Consumption of small amount of chilli enriches diet and considered as of minerals, vitamins and other food components (Farhad et al., 2010). Both green and dry chillies are one of the rich sources of Vitamin $\mathrm{A}$ and $\mathrm{C}$ and the seeds contain traces of starch. Capsaicin and oleoresin are also used in many pharmaceutical preparations like pain balms, vapor linements, skin ointments and ointment for cold, sore throat, chest congestion etc. India is now one of the leading chilli producing and is the largest exporter countries in the world and at present 2.5 to $3.0 \%$ of the total production of India is exported. In spite of its nutritive, commercial, pharmaceutical as well export values, India is 
still lagging far behind to attain the average productivity of chilli in the world (14.4 $t$ green chilli/ha). Huge advantage of wide cultivation and presence of huge quantum of genetic diversity could not so far been capitalized. Therefore, much concerted efforts are necessary to improve its yield and yield attributes. Chilli cultivars are generally distinguished on the basis of morphological traits and have a wide variability of botanical characteristics. The existence of variability in a particular trait is an important prerequisite for its heritable improvement. High yield with good quality is the most important objective in chilli breeding. The progress in breeding for yield and its contributing characters of any crop is polygenically controlled, environmentally influenced and determined by the magnitude and nature of their genetic variability (Wright,1935 and Fisher, 1981). The magnitude of heritable variation of the genetic components is very important to understand their genetic constitution which has a close bearing on its response to selection. Study of correlation between different quantitative characters provides an idea of association that could be effectively utilized in selecting a better plant type in chilli breeding programme. Correlation in grouping with path analysis would give a better insight into cause and effect relationship between different pairs of characters (Jayasudha and Sharma, 2010). Partitioning of total correlation into direct and indirect effect by path analysis helps in making the selection more effective (Priya and Joel, 2009). A significant association suggests that such characters could be improved simultaneously. However, such an improvement depends on phenotypic correlation, additive variance and heritability (Hayes et al., 1955). It is necessary to have a good knowledge of those characters that have significant association with yield because the characters can be used to direct selection criteria or indices to enhance performances of varieties in a new plant population Considering the importance of chilli on these aspects the present investigation was taken up to evaluate chilli germplasm to identify genotypes with higher yield by study these genetic parameters as well as to determine interrelationship among the characters and their direct and indirect effects on yield which would be utilized for further improvement of chilli through appropriate breeding programs.

\section{Materials and Methods}

The field experiments were carried out at Central Research Farm, Gayeshpur, Bidhan Chandra Krishi Viswavidyalaya, Nadia, West Bengal, lying at $22^{0} 57^{\prime} \mathrm{N}$ latitude and $88^{\circ} 20^{\prime} \mathrm{E}$ longitude with an average altitude of $9.75 \mathrm{~m}$ above the mean sea level. It comes under Gangetic new alluvial plain of sandy loam soil with neutral to slight acidity. The experimental site is situated under subtropical humid region with an average temperature range of $25-37{ }^{0} \mathrm{C}$ during summer to $12-25{ }^{0} \mathrm{C}$ during winter months. Field experiments were carried out over the period of three season and Twenty four genotypes (Table 1) were evaluated in Randomized Complete Block Design with three replications. Each plot consisted of 20 plants spaced by $50 \mathrm{~cm}$ x $50 \mathrm{~cm}$. Standard crop management practices and plant protection measures were taken time to time. Ten randomly selected plants from each replication were taken to record the following quantitative observations. Quantitative character were recorded such as plant height, plant canopy widtht, primary branches, plant ${ }^{1}$, days to flowering, fruits plant ${ }^{-1}$, fruit length $(\mathrm{cm})$, fruit grith $(\mathrm{cm})$, seed fruit ${ }^{-1}$, fruit weight (gm), pericarp thickness, fruit pedicel length $\mathrm{cm}$, pedicel/fruit ratio, dry weight of ripe fruit and fruit yield plant $^{-1}$. The genotypic and phenotypic coefficient of variation (GCV and PCV respectively) was calculated on the basis of formula given by Burton (1952). 
Heritability in broad sense was calculated according to the formula suggested by Jhonson et al., (1955). Genotypic correlation coefficients were estimated using the formula suggested by Singh and Chaudhury (1979), Johnson et al., (1955) and Al-Jibouri et al., (1958). Path coefficients analysis was done according to the method suggested by Dewey and $\mathrm{Lu}$ (1959). All the statistical analysis was carried out using Genres computer software.

\section{Results and Discussion}

\section{Genetic variability for yield components of chilli}

Analysis of variance revealed significant differences among the genotypes for almost all the characters except fruit length, fruit weight and pedicel/ fruit ratio (Table 1). The wide range of variation noticed in all the characters would offer scope of selection for improvement of desirable types. The extent of variability present in the genotypes was measured in terms of range, coefficient of variation, genotypic coefficient of variation (GCV), phenotypic coefficient of variation (PCV), broad sense heritability and genetic advance (GA). It was found that moderate to high range of variation was recorded in all characters, indicating better scope for improvement through selection. Mean growth, fruit characters and fruit yield of the 24 varieties (Table 2) showed quite a good genotypes among the varieties for almost all the 14 characters, indicating the suitability of the genotypes for carrying out the study of genetic variability for different characters. Fruit length is the most important character of chilli particularly for export purpose. Top five genotypes for long and attractive fruits were viz., AC-173 (10.47), AC-571 (10.45), Hyb3(2)-3 (10.22), AC-575 (9.9 cm) and Hyb$3(2)-1(9.06 \mathrm{~cm})$. Fruit yield appeared to a cumulative character of fruit number plant $^{-1}$, fruit length and fruit weight. The highest fruit yielding genotypes viz., Hyb-3(2)-2, Hyb3(2)-1, AC-571 had long fruit and of the other two genotypes, AC-573 had medium long fruit and AC-575 had medium fruit length. Hence, all these genotypes hold ample promise to be regarded as a promising variety for green chilli purpose.

The coefficient of variation estimated was low to moderate for plant height, plant canopy, days to first flowering, primary branches plant ${ }^{-1}$ and pedicel length and moderately high to very high for fruits plant ${ }^{-1}$, fruit length, fruit girth, seeds fruit $^{-1}$, pericarp thickness, pedicel: fruit ratio and both green and ripe fruit yield plant $^{-1}$ (Table 3). Both phenotypic coefficient of variation (PCV) and genotypic coefficient of variation (GCV) showed wide differences for the characters particularly, fruit girth, fruit weight and pericarp which indicated marked genotypefruit harvest interaction for the expression of these characters. Highest mean genotypic coefficient of variation was recorded for fruit yield plant $^{-1}$ followed by fruits plant ${ }^{-1}$ and fruit weight. In the present investigation fruit yield plant $^{-1}$, fruits plant ${ }^{-1}$, fruit length, fruit girth and fruit weight showed high GCV. The high broad sense heritability ( $80 \%$ and above) was recorded for the characters namely, fruit yield plant $^{-1}$, plant height, plant canopy, days to first following, fruits plant ${ }^{-1}$, seeds fruit ${ }^{-1}$ and pedicel length (Table 3). High genetic advance as percentage of mean considering for fruit yield plant $^{-1}(86.80 \%)$, number of fruits plant $^{-1}(72.48 \%)$, fruit girth $(70.53 \%)$ and seeds fruit ${ }^{-1}(62.71 \%)$.

\section{Character association and path co-efficient analysis}

Selection of a character for its improvement may simultaneously lead to selection of the associated characters. Therefore, it is essential to understand the inter-relationship among 
different characters so that improvement of the targeted character does not carry with it the non-targeted characters rather desirable characters could be simultaneously included which may lead to ultimate success on breeding programme. Genotypic (G) and phenotypic (P) correlation coefficients among the pair of yield contributing characters pertaining to chilli (Table 4) have been presented. The fruit yield plant $^{-1}$ was significantly and positively correlated consistently with days to first flowering $(\mathrm{rP}=$ $0.492)$, number of fruits plant ${ }^{-1}(\mathrm{rP}=0.694)$ and seeds fruit ${ }^{-1}(\mathrm{rP}=0.627)$. Fruit length was significantly and positively correlated with fruit girth and fruit weight indicating that with fruit length may increase concomitantly with the increase in fruit girth and weight. Number of fruits plant $^{-1}$ was positively and significantly correlated with seeds fruit ${ }^{-1}$.

The path coefficient analysis using phenotypic correlation coefficient among pair of characters depicting direct and indirect effect on yield chilli did not change considerably with the harvest pattern of the fruits (Table 5). Highest positive direct effect on yield was registered by fruit weight (0.824), number of fruits plant ${ }^{-1}(0.630)$ followed by primary branches plant ${ }^{-1}(0.435)$ and plant canopy (0.147). Of these three characters, only fruits plant ${ }^{-1}$ had significant and positive correlation with fruit yield which indicated that linear correlation did not always suggest that the character would exert high and positive direct effect on the dependent variable, yield in this investigation. From the study of character association, combining correlation and path co-efficient, the characters namely, fruit weight, number of fruits plant ${ }^{-1}$, primary branches plant ${ }^{-1}$ and plant canopy were the most important selection criteria for improving fruit yield of chilli.

The wide range of variation noticed in all the characters would offer scope of selection for improvement of desirable types. Significant variations in the mentioned characters in chilli genotypes were earlier reported by Gogoi et al., (2002), Chowdhary and Samadia (2004) and many other workers. In the present investigation fruit yield plant ${ }^{-1}$, fruits plant ${ }^{-1}$, fruit length, fruit girth and fruit weight showed high GCV and this finding was supported by Choudhary and Samadia (2004) and Rani et al. (1996). The high broad sense heritability ( $80 \%$ and above) was recorded for the characters namely, fruit yield plant ${ }^{-1}$, plant height, plant canopy, days to first following, fruits plant ${ }^{-1}$, seeds fruit ${ }^{-1}$ and pedicel length (Table 3) which confirmed the earlier findings of Choudhary and Samadia (2004), Rani et al., (1996). High estimates of broad sense heritability in the quantitative characters has been found to be useful from plant breeders view point as this would enable him to base his selection on the phenotypic performance.

High genetic advance as percentage of mean considering for fruit yield plant ${ }^{-1}(86.80 \%)$, number of fruits plant ${ }^{-1}(72.48 \%)$, fruit girth $(70.53 \%)$ and seeds fruit ${ }^{-1}(62.71 \%)$ were in also conformity with the findings of Chowdhary and Samadia (2004). Johnson et al., (1955) had suggested that heritability estimates along with genetic gain is usually more helpful than the heritability alone in predicting the resultant effect for selecting best individuals. So these two genetic parameters must be considered together to predict the expected genetic progress possible through selection. In the present investigation, moderate GCV coupled with high broad sense heritability and moderately high genetic advance was registered in three characters namely fruit yield plant ${ }^{-1}$, number of fruits plant $^{-1}$, fruit girth and seeds fruit ${ }^{-1}$. Report on the combination of these genetic variability estimates for these characters was supported by earlier finding of Acharyya et al., (2003), Manju and Sreelathakumary (2002). 
Int.J.Curr.Microbiol.App.Sci (2017) 6(3): 2002-2012

Table.1 Analysis of variance for different component characters of chilli

\begin{tabular}{lccc}
\hline Characters & Genotypes & $\begin{array}{c}\text { Mean sum of squares } \\
\text { Replication }\end{array}$ & Error \\
\hline Plant height & $112.648^{* *}$ & 3.51 & 5.69 \\
Plant canopy(N-S) & $76.79^{* *}$ & 0.29 & 4.49 \\
Plant canopy(E-W) & $80.44^{* *}$ & 0.66 & 5.40 \\
Days to first flowering & $73.86^{* *}$ & 1.52 & 4.69 \\
Primary branches/ plant & $5.74^{* *}$ & 0.41 & 0.64 \\
Fruits/plant & $824.66^{* *}$ & 51.09 & 57.28 \\
Fruit length & $76.60 \mathrm{NS}$ & 29.22 & 39.03 \\
Fruit girth & $5.76^{* *}$ & 0.57 & 0.55 \\
Seeds/fruit & $1346.50^{* *}$ & 7.98 & 57.47 \\
Fruit weight & $58.50 \mathrm{NS}$ & 49.03 & 46.45 \\
Pericarp thickness & $0.61^{* *}$ & 0.18 & 0.18 \\
Pedicel length & $0.36^{* *}$ & 0.02 & 0.03 \\
Pedicel:fruit ratio & $0.13 \mathrm{NS}$ & 0.17 & 0.14 \\
Fruit yield & $31131.01^{* *}$ & 1924.50 & 1832.55 \\
\hline
\end{tabular}


Int.J.Curr.Microbiol.App.Sci (2017) 6(3): 2002-2012

Table.2 Mean of different yield component characters of chilli

\begin{tabular}{|c|c|c|c|c|c|c|c|c|c|c|c|c|c|c|}
\hline Genotype & $\begin{array}{c}\text { Plant } \\
\text { height } \\
(\mathrm{cm})\end{array}$ & $\begin{array}{c}\text { Plant } \\
\text { Canopy } \\
(\mathrm{N}-\mathrm{S}) \\
(\mathrm{cm}) \\
\end{array}$ & $\begin{array}{c}\text { Plant } \\
\text { canopy } \\
(\mathrm{E}-\mathrm{W}) \\
(\mathrm{cm}) \\
\end{array}$ & $\begin{array}{c}\text { Days to } \\
\text { first } \\
\text { floweri } \\
\text { ng } \\
\end{array}$ & $\begin{array}{c}\text { Primary } \\
\text { branches/ } \\
\text { plant }\end{array}$ & $\begin{array}{c}\text { Fruits/ } \\
\text { plant }\end{array}$ & $\begin{array}{c}\text { Fruit } \\
\text { length } \\
(\mathrm{cm})\end{array}$ & $\begin{array}{l}\text { Fruit } \\
\text { girth } \\
(\mathrm{cm})\end{array}$ & $\begin{array}{c}\text { Seeds/ } \\
\text { fruit }\end{array}$ & $\begin{array}{c}\text { Fruit } \\
\text { weight } \\
\text { (g) }\end{array}$ & $\begin{array}{c}\text { Pericar } \\
\text { p } \\
\text { thickne } \\
\text { ss }(\mathbf{m m}) \\
\end{array}$ & $\begin{array}{c}\text { Pedicel } \\
\text { length } \\
(\mathrm{cm})\end{array}$ & $\begin{array}{c}\text { Pedicel } \\
\text { : } \\
\text { fruit } \\
\text { ratio }\end{array}$ & $\begin{array}{c}\text { Fruit } \\
\text { Yield/plant } \\
\text { (g) }\end{array}$ \\
\hline Hyb-3(2)-3 & 48.10 & 44.37 & 44.30 & 51.30 & 3.80 & 41.53 & 10.22 & 5.12 & 38.44 & 8.31 & 3.36 & 3.12 & 1.27 & 244.22 \\
\hline Hyb 3(2)-1 & 39.27 & 39.43 & 31.27 & 50.27 & 6.33 & 54.13 & 9.06 & 4.53 & 96.33 & 8.47 & 2.18 & 2.53 & 0.28 & 428.67 \\
\hline KDCS 810 & 38.83 & 36.33 & 33.13 & 38.83 & 10.37 & 37.17 & 6.82 & 3.68 & 78.50 & 6.45 & 1.96 & 2.59 & 0.39 & 117.61 \\
\hline Pant C-1 & 38.23 & 29.90 & 32.20 & 35.43 & 6.23 & 72.60 & 5.17 & 2.91 & 77.37 & 4.76 & 1.57 & 2.72 & 0.54 & 228.30 \\
\hline AC571 & 30.23 & 32.23 & 37.23 & 45.43 & 7.17 & 50.82 & 10.45 & 3.96 & 75.63 & 8.88 & 1.45 & 2.82 & 0.27 & 343.20 \\
\hline $\mathrm{AC} 173$ & 37.23 & 39.23 & 33.13 & 33.43 & 6.63 & 58.87 & 10.47 & 3.52 & 65.30 & 5.26 & 1.97 & 3.15 & 0.30 & 254.98 \\
\hline AC501 & 38.13 & 36.83 & 37.43 & 34.70 & 7.20 & 34.47 & 5.66 & 2.48 & 38.60 & 5.44 & 1.63 & 2.86 & 0.52 & 117.65 \\
\hline BC-4 & 46.23 & 35.27 & 29.63 & 34.23 & 7.83 & 16.30 & 3.92 & 3.01 & 43.33 & 7.31 & 1.77 & 2.47 & 0.68 & 74.76 \\
\hline BCC 54 & 47.93 & 41.43 & 29.90 & 38.23 & 8.67 & 20.27 & 7.74 & 2.98 & 53.83 & 6.84 & 1.41 & 3.47 & 0.45 & 81.85 \\
\hline AC 587 & 43.17 & 40.17 & 40.80 & 36.87 & 10.23 & 63.57 & 8.13 & 2.67 & 76.13 & 5.50 & 1.50 & 3.18 & 0.40 & 249.57 \\
\hline HP25 & 29.27 & 35.10 & 40.40 & 37.40 & 5.47 & 13.83 & 8.28 & 3.09 & 31.40 & 8.50 & 2.36 & 3.15 & 0.38 & 135.72 \\
\hline HP 29 & 29.13 & 30.20 & 27.40 & 37.80 & 6.57 & 19.27 & 8.36 & 3.11 & 37.83 & 8.64 & 2.42 & 2.81 & 0.34 & 124.19 \\
\hline HP 27 & 27.23 & 34.43 & 27.27 & 33.27 & 7.20 & 17.40 & 8.97 & 1.67 & 42.80 & 7.25 & 2.46 & 2.79 & 0.31 & 157.97 \\
\hline AC 576 & 39.87 & 38.17 & 34.73 & 40.30 & 8.20 & 44.30 & 7.48 & 0.53 & 51.83 & 6.79 & 1.85 & 2.34 & 0.32 & 210.52 \\
\hline PBC 374 & 32.70 & 26.70 & 28.73 & 42.23 & 6.43 & 26.20 & 8.15 & 1.41 & 43.60 & 7.63 & 2.43 & 2.07 & 0.26 & 161.28 \\
\hline AC 573 & 45.27 & 36.30 & 39.77 & 38.57 & 6.97 & 64.27 & 9.62 & 3.13 & 78.63 & 5.68 & 1.83 & 3.42 & 0.36 & 305.11 \\
\hline AC-585 & 36.43 & 33.23 & 35.43 & 40.83 & 6.70 & 55.80 & 6.81 & 3.45 & 61.60 & 7.56 & 1.59 & 2.57 & 0.38 & 246.31 \\
\hline AC-575 & 40.17 & 41.17 & 38.83 & 38.57 & 7.17 & 42.70 & 9.90 & 4.38 & 90.13 & 8.85 & 2.44 & 2.82 & 0.29 & 294.43 \\
\hline KA-2-1 & 32.23 & 28.23 & 24.83 & 37.17 & 8.30 & 48.47 & 8.32 & 3.68 & 112.80 & 7.98 & 1.86 & 3.20 & 0.39 & 273.44 \\
\hline Hyb 3(2)-2 & 42.13 & 35.33 & 36.27 & 44.13 & 7.30 & 48.70 & 9.83 & 4.32 & 85.43 & 9.58 & 1.75 & 2.74 & 0.28 & 437.36 \\
\hline BCC 62 & 41.97 & 23.77 & 28.77 & 37.93 & 6.63 & 34.57 & 8.32 & 3.56 & 75.60 & 5.65 & 1.81 & 3.12 & 0.38 & 157.27 \\
\hline LCA 235 & 42.13 & 30.43 & 27.27 & 32.83 & 6.70 & 46.27 & 5.49 & 2.94 & 55.87 & 6.39 & 1.71 & 2.79 & 0.53 & 150.18 \\
\hline AC 615 & 44.47 & 39.27 & 34.87 & 34.27 & 8.43 & 28.47 & 6.42 & 2.98 & 67.43 & 6.45 & 1.55 & 3.10 & 0.49 & 89.90 \\
\hline AC 574 & 33.17 & 35.81 & 37.93 & 35.20 & 7.47 & 46.23 & 8.71 & 4.17 & 56.37 & 6.87 & 2.09 & 3.29 & 0.38 & 280.26 \\
\hline $\operatorname{S.Em}( \pm)$ & 1.95 & 1.73 & 1.91 & 1.77 & 0.65 & 6.81 & 5.11 & 0.61 & 6.91 & 5.57 & 0.34 & 0.15 & 0.30 & 34.95 \\
\hline C.D. $(P=0.05)$ & 3.94 & 3.49 & 3.86 & 3.58 & 1.31 & 13.76 & 10.32 & 1.23 & 13.96 & 11.25 & 0.69 & 0.30 & 0.61 & 70.60 \\
\hline
\end{tabular}


Int.J.Curr.Microbiol.App.Sci (2017) 6(3): 2002-2012

Table.3 Genetic variability parameters for different yield component characters of chilli

\begin{tabular}{|c|c|c|c|c|c|c|c|}
\hline $\begin{array}{r}\text { Component of } \\
\text { variation }\end{array}$ & Mean & $\begin{array}{c}\text { Coefficient of } \\
\text { variation(C.V.) } \\
\%\end{array}$ & $\operatorname{PCV}(\%)$ & GCV $(\%)$ & Heritability (\%) & $\begin{array}{c}\text { Genetic } \\
\text { Advance (GA) }\end{array}$ & $\begin{array}{c}\text { Genetic } \\
\text { Advance } \\
(\% \text { of Mean })\end{array}$ \\
\hline Plant height & 38.48 & 6.19 & 16.71 & 15.52 & 862 & 11.42 & 29.68 \\
\hline Plant canopy(N-S) & 35.14 & 6.03 & 15.22 & 13.97 & 843 & 9.28 & 26.41 \\
\hline Plant canopy(E-W) & 33.81 & 6.87 & 16.31 & 14.79 & 823 & 9.34 & 27.62 \\
\hline Days to first flowering & 38.72 & 5.59 & 13.60 & 12.40 & 83.1 & 9.02 & 23.30 \\
\hline Primary branches/ plant & 7.25 & 11.02 & 21.09 & 17.98 & 72.7 & 2.29 & 31.59 \\
\hline Fruits/plant & 41.09 & 18.41 & 43.06 & 3892 & 81.7 & 29.78 & 72.48 \\
\hline Fruit length & 8.89 & 30.29 & 80.79 & 39.82 & 243 & 359 & 40.39 \\
\hline Fruit girth & 3.35 & 22.209 & 452 & 3937 & 759 & 236 & 70.53 \\
\hline Seeds/fruit & 63.95 & 11.8548 & 3451 & 3241 & 882 & 40.1 & 62.71 \\
\hline Fruit weight & 6.26 & 20.89 & 11358 & 3204 & 08.00 & 1.16 & 18.55 \\
\hline Pericarp thickness & 1.96 & 21.547 & 2893 & 193 & 445 & 052 & 26.58 \\
\hline Pedicel length & 2.88 & 6.337 & 13.16 & 1153 & 76.8 & 0.6 & 20.84 \\
\hline Pedicel:fruit ratio & 0.43 & 26.46 & 86.79 & 745 & 750 & 0.01 & 2.36 \\
\hline Fruit yield & 215.20 & 19.893 & 50.05 & 4592 & 842 & 1868 & 86.80 \\
\hline
\end{tabular}


Table.4 Genotypic (G) and Phenotypic (P) correlation of chilli for yield and its attributing traits

\begin{tabular}{|c|c|c|c|c|c|c|c|c|c|c|c|c|c|c|c|}
\hline Characters & & $\begin{array}{l}\text { Plant } \\
\text { heigh } \\
\text { t }\end{array}$ & $\begin{array}{l}\text { Plant } \\
\text { canop } \\
\text { y } \\
(\mathrm{N}-\mathrm{S}) \\
\end{array}$ & $\begin{array}{l}\text { Plant } \\
\text { Canopy } \\
(\text { E-W) }\end{array}$ & $\begin{array}{l}\text { Days to } \\
\text { first } \\
\text { flowerin } \\
\mathrm{g}\end{array}$ & $\begin{array}{l}\text { Primary } \\
\text { branche } \\
\text { s/ plant }\end{array}$ & $\begin{array}{l}\text { Fruits/ } \\
\text { plant }\end{array}$ & $\begin{array}{l}\text { Fruit } \\
\text { lengt } \\
\text { h }\end{array}$ & $\begin{array}{l}\text { Fruit } \\
\text { girth }\end{array}$ & $\begin{array}{l}\text { Seeds/fru } \\
\text { it }\end{array}$ & $\begin{array}{l}\text { Fruit } \\
\text { weigh } \\
\text { t }\end{array}$ & $\begin{array}{l}\text { Pericar } \\
\text { p } \\
\text { thicknes } \\
\text { s }\end{array}$ & $\begin{array}{l}\text { Pedicel } \\
\text { length }\end{array}$ & $\begin{array}{l}\text { Pedicel: } \\
\text { fruit } \\
\text { ratio }\end{array}$ & $\begin{array}{l}\text { Fruit } \\
\text { yield }\end{array}$ \\
\hline \multirow[t]{2}{*}{$\begin{array}{l}\text { Plant } \\
\text { height }\end{array}$} & $\mathbf{P}$ & 1.000 & $\begin{array}{l}0.497 * \\
*\end{array}$ & 0.307 & 0.204 & 0.089 & 0.159 & 0.169 & 0268 & 0.119 & 0073 & -0.125 & 0.179 & 0301 & -0055 \\
\hline & $\mathbf{G}$ & 1000 & 0451 & 0235 & 0.087 & 0.150 & 0205 & 0278 & 0313 & 0.127 & 0,082 & -0228 & 0233 & 3201 & -0048 \\
\hline Plant & $\mathbf{P}$ & & 1.000 & $0.644^{*}$ * & 0.298 & 0.053 & 0.046 & 0307 & 0320 & $-0,062$ & 0241 & 0.178 & 0202 & 0212 & 0,097 \\
\hline $\begin{array}{l}\text { canopy } \\
\text { (N-S }\end{array}$ & $\mathbf{G}$ & & 1000 & 0.597 & 0.177 & 0.127 & 0.075 & 0563 & 0363 & -0082 & 0698 & 0.191 & 0300 & 2020 & 0.148 \\
\hline Plant & $\mathbf{P}$ & & & 1.000 & 0.385 & -0.174 & 0251 & 0357 & 0390 & $-00 \pi 7$ & 0284 & 0.179 & 0241 & 0201 & 0257 \\
\hline $\begin{array}{l}\text { Canopy } \\
\text { (E-W) }\end{array}$ & G & & & 1.000 & 0.300 & -0.157 & 0324 & 0.670 & OA51 & -0.108 & 0877 & 0.174 & 0307 & 1861 & 034 \\
\hline Days to & $\mathbf{P}$ & & & & 1.000 & -0.347 & 0.144 & OA48** & $050 B^{* *}$ & 0.161 & 0.414 & 0337 & -0215 & 0.156 & $0492^{* * *}$ \\
\hline $\begin{array}{l}\text { first } \\
\text { flowering }\end{array}$ & G & & & & 1000 & -0.393 & 0.199 & 0897 & 0600 & 0.187 & 1380 & 0446 & -0231 & 1276 & 0,62 \\
\hline Primary & $\mathbf{P}$ & & & & & 1.000 & 0.135 & $-0592^{* *}$ & $-0502^{* *}$ & 0365 & $-0.638^{* * k}$ & $0603^{* * k}$ & -0.131 & $-0.492^{* * k}$ & -0064 \\
\hline $\begin{array}{l}\text { branches/ } \\
\text { plant }\end{array}$ & G & & & & & 1000 & -0013 & -0531 & -0390 & 0324 & -0913 & -0598 & -0028 & -0801 & -0265 \\
\hline Fruits/ & $\mathbf{P}$ & & & & & & 1.000 & -0.179 & 0039 & $0643^{* * k}$ & -0334 & -0382 & 0.190 & -0308 & $0694 * *$ \\
\hline plant & G & & & & & & 1.00 & 0360 & 0293 & 0599 & 0220 & -0230 & 0,042 & 1238 & 0666 \\
\hline Fruit & $\mathbf{P}$ & & & & & & & 1.000 & $0.771^{* *}$ & -0238 & $0951^{* *}$ & $0.732^{* 2 k}$ & -0048 & $0806^{* 2 *}$ & -0007 \\
\hline length & $\mathbf{G}$ & & & & & & & 1000 & 0.827 & -0032 & 0964 & $0.70 B$ & 057 & -0.070 & 0602 \\
\hline \multirow[t]{2}{*}{ Fruit girth } & $\mathrm{P}$ & & & & & & & & 1000 & 0083 & $0.709^{* * *}$ & $0529^{12 k}$ & 0.170 & $0.619^{* k}$ & 0231 \\
\hline & $\mathrm{G}$ & & & & & & & & 1.000 & 0259 & 1027 & 0408 & 0442 & 2107 & 0488 \\
\hline \multirow[t]{2}{*}{ Seeds/fruit } & $\mathrm{P}$ & & & & & & & & & 1000 & -0325 & -0359 & 0.181 & -0372 & $0627^{* * *}$ \\
\hline & $\mathrm{G}$ & & & & & & & & & 1000 & -0234 & -0312 & 0089 & -1.184 & 0617 \\
\hline Fruit & $\mathrm{P}$ & & & & & & & & & & 1000 & $0.773^{* * *}$ & -0.183 & $0852^{* * *}$ & -0057 \\
\hline weight & G & & & & & & & & & & 1.000 & 1.108 & 0.686 & -3468 & 0938 \\
\hline Pericarp & $\mathrm{P}$ & & & & & & & & & & & 1000 & -0212 & $0559 \%$ & -0084 \\
\hline thickness & $\mathrm{G}$ & & & & & & & & & & & 1000 & 0000 & -0.133 & 0.159 \\
\hline Pedicel & $\mathrm{P}$ & & & & & & & & & & & & 1.000 & -0.147 & 0080 \\
\hline length & $\mathrm{G}$ & & & & & & & & & & & & 1000 & 2799 & -0051 \\
\hline Pedicel: & $\mathrm{P}$ & & & & & & & & & & & & & 1000 & -0338 \\
\hline \multirow[t]{2}{*}{ fruit ratio } & G & & & & & & & & & & & & & 1.000 & -0273 \\
\hline & $\mathrm{G}$ & & & & & & & & & & & & & & $0 A 34$ \\
\hline \multirow[t]{2}{*}{ Fruit yield } & $\mathrm{P}$ & & & & & & & & & & & & & & 1.000 \\
\hline & $\mathrm{G}$ & & & & & & & & & & & & & & 1000 \\
\hline
\end{tabular}


Table.5 Phenotypic path coefficient analysis for chilli yield as dependent variable

\begin{tabular}{|c|c|c|c|c|c|c|c|c|c|c|c|c|c|c|c|}
\hline Characters & Type & $\begin{array}{l}\text { Plant } \\
\text { height }\end{array}$ & $\begin{array}{l}\text { Plant } \\
\text { canopy } \\
(\mathrm{N}-\mathrm{S})\end{array}$ & $\begin{array}{l}\text { Plant } \\
\text { Canopy } \\
(\text { E-W) } \\
\end{array}$ & $\begin{array}{l}\text { Days to } \\
\text { first } \\
\text { flowering }\end{array}$ & $\begin{array}{l}\text { Primary } \\
\text { branches/ } \\
\text { plant }\end{array}$ & $\begin{array}{l}\text { Fruits/ } \\
\text { plant }\end{array}$ & $\begin{array}{l}\text { Fruit } \\
\text { length }\end{array}$ & $\begin{array}{l}\text { Fruit } \\
\text { girth }\end{array}$ & Seeds/fruit & $\begin{array}{l}\text { Fruit } \\
\text { weight }\end{array}$ & $\begin{array}{l}\text { Pericarp } \\
\text { thickness }\end{array}$ & $\begin{array}{l}\text { Pedicel } \\
\text { length }\end{array}$ & $\begin{array}{l}\text { Pedicel: } \\
\text { fruit } \\
\text { ratio }\end{array}$ & $\begin{array}{l}\text { Fruit } \\
\text { yield }\end{array}$ \\
\hline Plant height & Ripe & 0288 & 0.073 & 0.012 & 0.089 & 0.006 & 0.100 & 0.143 & 0.000 & 0.032 & 0.060 & 0.004 & 0,029 & 0.015 & -0.055 \\
\hline $\begin{array}{l}\text { Plant } \\
\text { canopy } \\
\text { (N-S) }\end{array}$ & Ripe & 0.143 & 0.147 & 0.026 & 0.130 & 0.003 & 0.029 & 0260 & 0.001 & 0.017 & 0.198 & 0.005 & 0.032 & 0.004 & 0.097 \\
\hline $\begin{array}{l}\text { Plant } \\
\text { Canopy } \\
\text { (E-W) }\end{array}$ & Ripe & 0.088 & 0.095 & 00040 & 0.167 & 0.011 & 0.158 & 0302 & 0,001 & 0.021 & 0234 & 0.005 & 0.039 & 0,000 & 0257 \\
\hline $\begin{array}{l}\text { Days to first } \\
\text { flowering }\end{array}$ & Ripe & 0.059 & 0.044 & 0.016 & $\mathbf{0 4 3 5}$ & 0.022 & 0,090 & 0379 & 0,001 & 0.043 & 0341 & 0.010 & 0.034 & 0.007 & 0.492 \\
\hline $\begin{array}{l}\text { Primary } \\
\text { branches/ } \\
\text { plant }\end{array}$ & Ripe & 0.026 & 0,008 & 0.007 & 0.151 & 0064 & 0,085 & 0500 & 0.001 & 0.098 & 0526 & 0.018 & 0.021 & 0.000 & -0.064 \\
\hline $\begin{array}{l}\text { Fruits/ } \\
\text { plant }\end{array}$ & Ripe & 0.046 & 0.007 & 0.010 & 0.062 & 0.009 & 0630 & 0.151 & 0.000 & 0.173 & 0275 & 0.012 & 0,031 & 0.009 & 0.694 \\
\hline Fruit length & Ripe & 0.049 & 0.045 & 0.014 & 0.195 & 0.038 & 0.113 & 0845 & 0.001 & 0.064 & 0.784 & 0.022 & 0.008 & 0.003 & -0.007 \\
\hline Fruit girth & Ripe & 0.077 & 0.047 & 0.016 & 0219 & 0.032 & 0.025 & 0.652 & -0.002 & 0.022 & 0.584 & 0.016 & 0.027 & 0.005 & 0231 \\
\hline Seeds/fruit & Ripe & 0,034 & 0.009 & $0.00 B$ & 0.070 & 0.023 & 0.405 & 0201 & 0.000 & 0269 & 0.268 & 0.011 & 0.029 & 0.006 & 0.627 \\
\hline Fruit weight & Ripe & 0.021 & 0.035 & 0.011 & 0.180 & 0.041 & 0210 & 0.804 & 0.001 & 0.087 & 0824 & 0.024 & 0.029 & 0.004 & -0.057 \\
\hline $\begin{array}{l}\text { Pericarp } \\
\text { thickness }\end{array}$ & Ripe & 0.036 & 0.026 & 0.007 & 0.146 & 0.038 & 0241 & 0.619 & 0.001 & 0.097 & 0.637 & 0030 & 0.034 & 0,000 & -0.084 \\
\hline $\begin{array}{l}\text { Pedicel } \\
\text { length }\end{array}$ & Ripe & 0.052 & 0.030 & 0.010 & 0.093 & 0.008 & 0.120 & 0.041 & 0,000 & 0.049 & 0.151 & 0.006 & 0.160 & 0,001 & 0.080 \\
\hline $\begin{array}{l}\text { Pedicel: } \\
\text { fruit ratio }\end{array}$ & Ripe & 0.171 & 0.024 & 0.000 & 0.109 & 0.000 & 0210 & 0.109 & 0.000 & 0.058 & 0.118 & 0,000 & 0,006 & 0026 & 0.484 \\
\hline
\end{tabular}


Generally high GCV coupled with high broad sense heritability and genetic advance is attributable to additive gene action controlling the concerned characters (Panse, 1957), so early generation selection would be helpful for improving the characters like, fruit yield plant $^{-1}$, number of fruits plant ${ }^{-1}$, fruit girth and seeds fruit $^{-1}$. Very low heritability accompanied with very low to moderately low genetic advance was recorded for fruit length, fruit weight, pericarp thickness and pedicel: fruit ratio indicating less responsiveness of these characters to selection. Improvement of these characters needs selection over several successive years, preferably across locations and over different plantings because such association of genetic parameters may be attributed to non-additive gene action (Liang and Walter, 1998) and high genotype-environment interaction, hence, simple selection will not be rewarding.

The correlation coefficients at genotypic level were in general higher than phenotypic correlation values. Higher genotypic correlations than phenotypic ones might be due to modifying or masking effect of environment in the expression of these characters under study as explained by Nandpuri et al., (1973). Johnson et al., (1955) also reported that higher genotypic correlation than phenotypic correlation indicated an inherent association between various characters. The findings on the positive direct effect of the fruit weight $(0.824)$, number of fruits plant ${ }^{-1}(0.630)$ followed by primary branches plant ${ }^{-1}(0.435)$ and plant canopy (0.147) on yield were supported by earlier works of Jabeen et al., (1999), and Jose et al., (2002). Significance of these characters as important selection indices was also suggested by earlier workers such as Devi et al., (1999), Kataria et al., (1997), Rani (1996) and Das et al,. (2000).

In conclusion, among these genotype Hyb$3(2)-2$ one of the most promising one showed maximum fruit yield plant ${ }^{-1}$, fruit length, fruit girth and pericarp thickness. Genetic variability of fruit yield plant $^{-1}$, number of fruits plant $^{-1}$, fruit girth and seeds fruit ${ }^{-1}$ emerged as most reliable characters for selection because of their probable conditioning by the additive gene action. Moderate GCV coupled with high broad sense heritability and moderately high genetic advance was registered in three characters namely fruit yield plant $^{-1}$, number of fruits plant $^{-1}$, fruit girth and seeds fruit ${ }^{-1}$. From the study of character associations both correlation and path co-efficient, the characters, namely, fruit weight, number of fruits plant ${ }^{-1}$, primary branches plant $^{-1}$ and plant canopy were the most important selection criteria for improving yield of chilli.

\section{References}

Acharyya, P., Rajput, C.B.S., and Acharyya, P. 2003. Variability and correlation studies for different traits in capsicum with respect to leaf curl complex. Indian J. Hort. 60 : 381-87.

Al-Jibouri, H. A., Miller, A., and Robinson, H. F. 1958. Genotypic and environmental variation and correlation in upland cotton cross of interspecies origin. Agron. J. 50: 633-37.

Burton, W.G. 1957. The influence of sprout development at planting on subsequent growth and yield. The growth of potato. Proc. of Tenth Easter School in Agril. Sci., Univ. of Nottingham, 1963. Butter Worths, London. pp. 21-29.

Choudhary, B.S., and Samadia, D.K. 2004. Variability and character association in chilli land races and genotypes under arid environment. Indian J. Hort. 61 : 132-36.

Das, M,. Behra, T.K., and Singh, G. 2000. Correlation and path coefficient analysis in chilli. Indian J. Hort. 57: 157-59.

Devi, D.S., Arumugam, R., and Devi, D.S. 1999. Correlation and path co-efficient 
analysis in chilli (C. annuum L.). Crop Res. 17: 90-93.

Fisher, R.A. 1981. The correlation among relative on the supposition of Mendelian Inheritance. Trans. Royal Soc. Edinberg.

Gogoi, D., Gautam, B.P., and Gogoi, D. 2002. Variability, heritability and genetic advance in chilli. Agric. Sci. Digest. 22: 102-104.

Hayes, H.K., Forrest, R.I., and Smith, D.C. 1955. Methods of plant Breeding correlation and regression in relation to plant breeding. McGrow - Hill Company Inc. 2nd Ed. pp. 439-57.

Jabeen, N., Ahmed, N., Tanki, M.I., and Jabeen, N. 1999. Genetic variability in hot pepper (C. annuum L.). Appl. Bio. Res. 1: 87-89.

Jayasudha, S., and Sharma, D. 2010. Genetic parameters of variability, correlation and pathcoefficient for grain yield and physiological traits in Rice (Oryza sativa L.) under shallow lowland situation. Electronic J. Plant Breed. 1: 33-38

Johnson, H.W., Robinson, H.F., and Comstock, R.E. 1955. Estimation of genetic and environmental variability in soybean. Agron. J. 47:314-18.

Jose,,L., Khader, K.M.A., and Jose, L. 2002. Correlation and path co-efficient analysis in chilli. Capsicum Egg plant Newsletter. 21 : 56-59.

Kataria, G.J., Pandey, H.M., and Vaddaria, M.A. 1997. Genetic variability, heritability and genetic advance of various polygenic traits in capsicum. Gujarat Agric. Univ. Res. J. 22 : 18-21.
Liang, G.H.L., and Walter, T.H. 1968. Heritability estimates and gene effects for agronomic traits in grain sorghum (Surghum vulgare). Crop Sci. 8: 77-80.

Manju, P.R., and Sreelathakumary, I. 2002. Genetic variability, heritability and genetic advance in hot chilli (Capsicum chinense Jacq).). J. Trop. Agric. 40:4-6.

Nandpuri, K.S., Singh, S., and Lal, T. 1973. Studies on genetic variability dnd corrcolatlon of some economic characters in tomato. J. Res. Punjab Agrie. Univ. 10: 316 -321.

Panse, V.G. 1957. Genetics of quantitative characters in relation to plant breeding. Indian J. Genet. 28: 225-29.

Pawade, S.B., Sontake, M.B., Shinde, N.N., and Borikar, S.T. 1995. Studies on correlation and path analysis for some characters in local chilli (C. annuum L.) types form Nagpur district. PKV. Res. J. $19:$ :93-94.

Priya, A.A., and Joel, A.J. 2009. Grain yield response of rice cultivars under upland condition, Electronic J. Plant Breed. 1: 6-11.

Rani, P.U., and Singh, D.P. 1996. Variability, heretability and genetic advance in chilli (C. annuum L). J. Res. APAU. 24 : 1-8.

Singh, R.K., and Choudhary, B.D. 1979. Biometrical Methods in Quantitative Genetic Analysis. Revised ed. Kalyani Publishers, New Delhi. pp. 57.

Wright, S. 1935. The analysis of variance and correlations between relative with respect to deviations from an optimum. J. Genet. 30: 243-256.

\section{How to cite this article:}

Dipak Kumar Murmu, Bimal Das, Rakesh Yanzone and Ratul Barman. 2017. Assessment of Genetic Variability, Character Association and Path Coefficient of Some Quantitative Traits of Chilli. Int.J.Curr.Microbiol.App.Sci. 6(3): 2002-2012.

doi: https://doi.org/10.20546/ijcmas.2017.603.228 